Bull. Korean Math. Soc. 48 (2011), No. 1, pp. 115-127

DOI 10.4134/BKMS.2011.48.1.115

\title{
A CONCEPT UNIFYING THE ARMENDARIZ AND NI CONDITIONS
}

\author{
Young Chun, Young Cheol Jeon, Sungkyung Kang, Key Nyoung Lee, \\ AND YANG LEE
}

\begin{abstract}
We study the structure of the set of nilpotent elements in various kinds of ring and introduce the concept of $N R$ ring as a generalization of Armendariz rings and $N I$ rings. We determine the precise relationships between $N R$ rings and related ring-theoretic conditions. The Köthe's conjecture is true for the class of $N R$ rings. We examined whether several kinds of extensions preserve the $N R$ condition. The classical right quotient ring of an $N R$ ring is also studied under some conditions on the subset of nilpotent elements.
\end{abstract}

\section{1. $N R$ rings}

Throughout this note every ring is associative with identity unless otherwise stated. Given a ring $R, N_{*}(R), N^{*}(R), J(R)$, and $N(R)$ denote the prime radical, the upper nilradical (i.e., sum of nil ideals), the Jacobson radical, and the set of all nilpotent elements in $R$, respectively. Note $N_{*}(R) \subseteq N^{*}(R) \subseteq$ $N(R)$ and $N^{*}(R) \subseteq J(R)$.

A ring is called reduced if it has no nonzero nilpotent elements. In the following we consider two kinds of generalizations of commutative rings. Due to Marks [20], a ring $R$ is called $N I$ if $N^{*}(R)=N(R)$. Note that $R$ is $N I$ if and only if $N(R)$ forms an ideal if and only if $R / N^{*}(R)$ is reduced. According to Birkenmeier et al. [5], a ring $R$ is called 2-primal if $N_{*}(R)=N(R)$. It is obvious that $R$ is 2-primal if and only if $R / N_{*}(R)$ is reduced. Marks [21] gave almost complete characterizations for 2-primal rings, with constructive delimiting examples. Note that a ring $R$ is reduced if and only if $R$ is nil-semisimple (i.e., $R$ has no nonzero nil ideals) and $N I$ if and only if $R$ is semiprime and 2-primal. It is obvious that 2-primal rings are $N I$, but the converse need not hold by Birkenmeier et al. [6, Example 3.3], Hwang et al. [13, Example 1.2], or Marks [20, Example 2.2]. If a ring $R$ is of bounded index of nilpotency, then

Received May 18, 2009.

2010 Mathematics Subject Classification. 16D25, 16N40.

Key words and phrases. NR ring, NI ring, Armendariz ring, matrix ring.

This study was supported by the Research Fund Program of Research Institute for Basic Sciences, Pusan National University, Korea, 2009, Project No. RIBS-PNU-2009-105. 
$R$ is $N I$ if and only if $R$ is 2-primal by [13, Proposition 1.4]. The upper triangular matrix rings over non-commutative reduced rings are typical examples of non-commutative 2-primal $(N I)$ rings.

Given a ring $R, R[x]$ denotes the polynomial ring with an indeterminate $x$ over $R$. For $f(x) \in R[x]$, let $C_{f(x)}$ denote the set of all coefficients of $f(x)$. Denote the $n$ by $n$ full matrix ring over $R$ by $\operatorname{Mat}_{n}(R)$ and the $n$ by $n$ upper (resp. lower) triangular matrix ring over $R$ by $U_{n}(R)$ (resp. $L_{n}(R)$ ). Use $e_{i j}$ for the matrix with $(i, j)$-entry 1 and elsewhere 0 .

When given a ring $R$ is reduced, Armendariz [3, Lemma 1] proved that $a_{i} b_{j}=0$ for all $i, j$ whenever $f(x) g(x)=0$, where $f(x)=\sum_{i=0}^{m} a_{i} x^{i}, g(x)=$ $\sum_{j=0}^{n} b_{j} x^{j}$ are in $R[x]$. Rege et al. [23] called a ring (not necessarily reduced) Armendariz if it satisfies the condition " $a_{i} b_{j}=0$ for all $i, j$ whenever $\left(\sum_{i=0}^{m} a_{i} x^{i}\right)\left(\sum_{j=0}^{n} b_{j} x^{j}\right)=0$. So reduced rings are Armendariz. If $R$ is an Armendariz ring, then $N(R)$ is a subring of $R$ by [2, Proposition 2.7 and Theorem $3.2]$. But here we prove that directly, applying the method in the proof of $[2$, Lemma 2.6].

Proposition 1.1. (1) Let $R$ be an Armendariz ring and $a^{m}=0, b^{m}=0$ for $a, b \in R$. Then $a^{\alpha} b^{\beta}(1 \leq \alpha, \beta \leq m-1), a-b \in N(R)$.

(2) If $R$ is an Armendariz ring, then $N(R)$ is a subring of $R$.

Proof. (1) Let $R$ be an Armendariz ring and $a, b \in N(R)$ with $a^{m}=0, b^{m}=0$. Let

$f(x)=\left(1+a x+\cdots+a^{m-1} x^{m-1}\right)(1-a x)(1-b x)\left(1+b x+b^{2} x^{2}+\cdots+b^{m-1} x^{m-1}\right)$.

Since $a^{m}=0=b^{m}, f(x)=1$ and $(a f(x))^{m}=a^{m}=0$, i.e.,

$\left(\left(a+a^{2} x+\cdots+a^{m-1} x^{m-1}\right)(1-a x)(1-b x)\left(1+b x+b^{2} x^{2}+\cdots+b^{m-1} x^{m-1}\right)\right)^{m}=0$.

Since $R$ is Armendariz, we get $\left(a^{\alpha} b^{\beta}\right)^{m}=0$ for $\alpha, \beta \in\{1, \ldots, m-1\}$.

Next we claim that $(a+b)^{2 m}=0$ by showing that every term is zero. Each term of $(a+b)^{2 m}$ is of the form $c_{i_{1}} c_{i_{2}} \cdots c_{i_{2 m}}$ with $c_{i_{j}}=a$ or $b$. Consider $g(x)=$ $(a+b x)^{2 m}$. Then every coefficient of $g(x)$ is a sum of $c=c_{i_{1}} c_{i_{2}} \cdots c_{i_{2 m}}$ 's. Note that $c$ contains at least $m$ occurrences of $a$ or $b$, say $c_{i_{j_{1}}}=c_{i_{j_{2}}}=\cdots=c_{i_{j_{m}}}=a$ with $1 \leq j_{1}<j_{2}<\cdots<j_{m} \leq 2 m$. Then

$$
c=c_{i_{1}} \cdots c_{i_{j_{1}-1}} a c_{i_{j_{1}+1}} \cdots c_{i_{j_{m}-1}} a c_{i_{j_{m}+1}} \cdots c_{i_{2 m}} .
$$

In the equation $(*)$, replacing each $c_{i_{j}}$ (for $i_{j} \notin\left\{j_{1}, j_{2}, \cdots, j_{m}\right\}$ ) by $f(x)$, we obtain

$$
f(x) \cdots f(x) a f(x) \cdots f(x) a f(x)=a^{m}=0 .
$$

Since $R$ is Armendariz, we get $c_{i_{1}} c_{i_{2}} \cdots c_{i_{2 m}}=0$ by taking proper coefficients of $f(x)$ 's. Use $-b$ in place of $b$ to obtain $(a-b)^{2 m}=0$.

Therefore $a^{\alpha} b^{\beta}(1 \leq \alpha, \beta \leq m-1), a-b \in N(R)$, entailing that $N(R)$ forms a subring of $R$.

(2) follows from (1) immediately. 
Commutative (hence $N I$ ) rings need not be Armendariz by [23, Example 3.2 ], and Armendariz rings need not be $N I$ by [2, Example 4.8].

In this note we will study the structure of rings whose nilpotent elements form subrings. Unifying the concepts of $N I$ rings and Armendariz rings, a ring $R$ (possibly without identity) will be called an $N R$ ring if $N(R)$ forms a subring of $R$. NI rings are clearly $N R$, and Armendariz rings are $N R$ by Proposition 1.1. But the converses are both irreversible by [23, Example 3.2] and [2, Example 4.8].

Considering the definition of $N R$ rings, one may suspect that $N(R) \subseteq J(R)$ for an $N R$ ring $R$. However the following example erases the possibility.

Example 1.2. Let $S=\mathbb{C}\{a, b\}$ be the free algebra with non-commuting indeterminates $a, b$ over $\mathbb{C}$, where $\mathbb{C}$ is the field of complex numbers. Let $I$ be an ideal of $S$ generated by $a^{2}$. Set $R=S / I$. Then $R$ is Armendariz (hence $N R$ ) by [2, Example 4.8] or [7, Example 9.3]. We coincide $a, b$ with their images in $R$ for simplicity. Notice that $N(R)$ is the subring of $R$ generated by

$$
\{\alpha a, \beta a r a \mid \alpha, \beta \in \mathbb{C}, r \in R\} .
$$

In spite of $a \in N(R), 1-b a$ is not invertible in $R$, entailing $a \notin J(R)$. This implies $N(R) \nsubseteq J(R)$.

Given a ring $R$ and an ideal $I$ of $R$, we denote $\{r \in R \mid r+I$ is regular in $R / I\}$ by $C(I)$.

Lemma 1.3. (1) The class of $N R$ rings is closed under subrings (possibly without identity).

(2) For any ring $A, \operatorname{Mat}_{n}(A)(n \geq 2)$ cannot be $N R$.

(3) Let $R$ be an $N R$ ring. If $C(0)=R \backslash N(R)$, then $R$ is $N I$.

(4) The class of NI rings is closed under subrings (possibly without identity).

(5) Let $R$ be a ring and $I$ a nil ideal of $R$. If $R / I$ is $N R$, then so is $R$.

(6) If $R$ is $N R$, then er $-r e \in N(R)$ for $e^{2}=e, r \in R$.

Proof. (1) Let $S$ be a subring of given a ring $R$. Then $N(S)=N(R) \cap S$. If $R$ is $N R, N(S)$ forms a subring of $S$. This implies that $S$ is $N R$.

(2) Since $n \geq 2, \operatorname{Mat}_{n}(R)$ contains two nilpotent elements $e_{12}, e_{21}$. But $e_{12}+e_{21} \notin N\left(\operatorname{Mat}_{n}(R)\right)$.

(3) Assume $C(0)=R \backslash N(R)$. Let $a \in N(R), r \in R$. By observing that $a r, r a$ are not regular, ar, ra $\in N(R)$ by assumption and so this implies that $N(R)$ is an ideal of $R$. Thus $R$ is $N I$.

(4) By $[13$, Proposition 2.4(2)].

(5) Let $\bar{R}=R / I$. If $a+I \in N(\bar{R})$, then $a \in N(R)$ since $I$ is nil, entailing $N(\bar{R}) \subseteq\{a+I \mid a \in N(R)\}$. The converse inclusion is evident, obtaining $N(\bar{R})=\{a+I \mid a \in N(R)\}$. Let $a, b \in N(R)$. Then $a+I, b+I \in N(\bar{R})$, and since $\bar{R}$ is $N R$ we get $(a-b)+I, a b+I \in N(\bar{R})$. The preceding equality yields $a-b, a b \in N(R)$.

(6) $a=e r(1-e), b=(1-e) r e \in N(R)$ implies $e r-r e=a-b \in N(R)$ when $R$ is $N R$. 
The class of $N R$ rings is not closed under factor rings. For example, let $R$ be the ring of quaternions with integer coefficients. Then $R$ is a domain and so $N R$. However for any odd prime integer $q$, the ring $R / q R$ is isomorphic to $\operatorname{Mat}_{2}\left(\mathbb{Z}_{q}\right)$ by the argument in $[10$, Exercise $2 \mathrm{~A}]$. Thus $R / q R$ is not $N R$.

Note. In Lemma 1.3(3) we consider only the condition " $C(0)=R \backslash N(R)$ " without the $N R$ condition. Then $a r, r a \in N(R)$ for $a \in N(R), r \in R$ by the proof of Lemma 1.3(3). Assume that $N(R)$ is of bounded index (of nilpotency) 2. Then for $a, b \in N(R),(a-b)^{4}=(a b+b a)^{2}=a b^{2} a+b a^{2} b=0$ since $a b, b a \in N(R)$. Thus $R$ is NI. Note $(a-b)^{2}=0$ with $a b+b a=0$.

Next assume that $N(R)$ is of bounded index (of nilpotency) $\geq 3$. Consider a sequence $(a, b, b a, b a a b, b a a b a b b a, \ldots)$ that is generated by $a, b \in N(R)$. The constructing rule is that a term is obtained from all that go before by interchanging the $a$ 's and the $b$ 's. Then by [22], there is no product $U$ of $a$ 's and $b$ 's such that $U^{3}$ occurs in the sequence. So in this case we do not know whether the condition " $C(0)=R \backslash N(R)$ " implies the $N I$ (or $N R$ ) condition.

Question. Let $R$ be a ring. If $N(R)$ is of bounded index (of nilpotency) $\geq 3$ and $C(0)=R \backslash N(R)$, then is $R$ an $N I$ (or $N R$ ) ring?

A ring $R$ is called directly finite if $a b=1$ implies $b a=1$ for $a, b \in R . N I$ rings and Armendariz rings are directly finite by [13, Proposition 2.7] and [18, Lemma 3.4(3)], respectively. So one may conjecture that $N R$ rings are directly finite.

Proposition 1.4. NR rings are directly finite.

Proof. We apply the proof of [13, Proposition 2.7]. Let $R$ be an $N R$ ring and assume on the contrary that $R$ is not directly finite. Then $R$ contains an infinite set of matrix units, say $\left\{e_{11}, e_{12}, e_{13}, \ldots, e_{21}, e_{22}, e_{23}, \ldots\right\}$, by $[9$, Proposition 5.5]. Since $N(R)$ forms a subring in $R$ and $e_{12}, e_{21} \in N(R), e_{11}=$ $e_{12} e_{21} \in N(R)$, a contradiction.

By Propositions 1.1 and 1.4, Armendariz rings and NI rings are directly finite.

A ring is called Abelian if every idempotent is central. Abelian rings are directly finite by a simple computation. Armendariz rings are Abelian by the proof of [1, Theorem 6] or [12, Corollary 8]. Armendariz rings are $N R$ by Proposition 1.1. So it is necessary to check the implications between $N R$ rings and Abelian rings. But they are independent of each other as follows.

Example 1.5. (1) Let $K$ be a field and $S=K\left\{a_{0}, a_{1}, a_{2}, a_{3}\right\}$ the free algebra with non-commuting indeterminates $a_{0}, a_{1}, a_{2}, a_{3}$ over $K$. Let $I$ be the ideal of $S$ generated by $a_{0} a_{1}, a_{2} a_{3}$. Set $R=S / I$ and we coincide $a_{0}, a_{1}, a_{2}, a_{3}$ with their images in $R$ for simplicity. It is easily checked that $R$ has only two idempotents 0 and 1 , entailing that $R$ is Abelian. Now consider $a_{1} a_{0}$ and $a_{3} a_{2}$. By the construction of $R, a_{1} a_{0}$ and $a_{3} a_{2}$ are nilpotent. But $a_{1} a_{0}+a_{3} a_{2} \notin N(R)$. So $R$ is not $N R$. 
(2) There exists an $N R$ ring but not Abelian as can be seen by $U_{2}(F)$ over a field $F$.

It is obvious that Köthe's conjecture (i.e., the sum of two nil left ideals is nil) holds for $N I$ rings.

Proposition 1.6. Let $R$ be an $N R$ ring. Then the Köthe's conjecture holds. Especially $N^{*}\left(\operatorname{Mat}_{n}(R)\right)=\operatorname{Mat}_{n}\left(N^{*}(R)\right)$ and $J(R[x])=N^{*}(R)[x]$.

Proof. Let $R$ be an $N R$ ring and $I, J$ be nil left ideals of $R$. Then $a+b \in N(R)$ for $a \in I, b \in J$ and so $I+J$ is nil. The other results are obtained by [24, Theorem 2.6.35].

One may suspect that if the Köthe's conjecture holds, then $R$ is $N R$. But it's not true. For example, let $F$ be a field and let $\operatorname{Mat}_{2}(F)$. Then by $[24$, Theorem 2.6.35], if the Köthe's conjecture holds, then $N^{*}\left(\operatorname{Mat}_{2}(F)\right)=\operatorname{Mat}_{2}\left(N^{*}(F)\right)=$ 0 . But clearly $N\left(\operatorname{Mat}_{2}(F)\right) \neq 0$, and $\operatorname{Mat}_{2}(F)$ is not $N R$ by Lemma $1.3(2)$.

Denote the center of a ring $R$ by $Z(R)$. If $N(R) \subseteq Z(R)$, then $N(R)$ is an ideal of $R$, obtaining that $R$ is $N I$ (hence $N R$ ). The following is compared with this result.

Proposition 1.7. If $R$ is a ring such that $N(R)$ is a commutative subset of $R$, then $R$ is $N R$.

Proof. Let $a, b \in N(R)$. Then if $N(R)$ is commutative, $a-b \in N(R)$ and $a b \in N(R)$ and so $R$ is $N R$.

Consider $R=U_{2}(A)$ over a reduced ring $A$. Then $N(R)=\left(\begin{array}{ll}0 & A \\ 0 & 0\end{array}\right)$ is a commutative subset of $R$ since $x y=0=y x$ for $x, y \in N(R)$. So $R$ is $N R$ by the Proposition 1.7. Letting $I=\left(\begin{array}{ll}0 & A \\ 0 & 0\end{array}\right), I$ is a nil ideal of $R$ such that $R / I \cong A \oplus A$ is reduced (hence $N R$ ). $R$ is $N R$ also by Lemma 1.3(5).

A ring $R$ is called (von Neumann) regular if for each $a \in R$ there exists $x \in R$ such that $a=a x a$.

Proposition 1.8. Given a regular ring $R$ the following conditions are equivalent:

(1) $R$ is Armendariz;

(2) $R$ is $N I$;

(3) $R$ is $N R$;

(4) $R$ is reduced;

(5) $R$ is 2-primal;

(6) $R$ is Abelian.

Proof. Since $R$ is regular, $J(R)=0$ by [9, Corollary $1.2(\mathrm{c})]$ and so the equivalences of the conditions $(2),(4),(5)$ are obtained. [9, Theorem 3.2] gives the equivalences of the conditions (4) and (6). (1) implies (6) by [12, Corollary 8]. (4) implies (1) by [3, Lemma 1]. (2) implies (3) trivially. (3) implies (6) by [15, Theorem 1.8]. 
A ring $R$ is called $\pi$-regular if for each $a \in R$ there exist a positive integer $n$, depending on $a$, and $b \in R$ such that $a^{n}=a^{n} b a^{n}$. The Jacobson radicals of $\pi$-regular rings are nil, comparing with that regular rings are semiprimitive. Regular rings are clearly $\pi$-regular. However the preceding results need not hold on $\pi$-regular rings. $U_{n}(D)(n \geq 2$ and $D$ is a division ring) is $\pi$-regular and $N R$, but it is neither regular nor reduced.

\section{Examples of $N R$ rings}

In this section we consider several kinds of ring extensions over $N R$ rings, and examine them to be $N R$. We see typical examples of $N R$ rings in the following.

Theorem 2.1. For a ring $R$ and $n \geq 2$, the following conditions are equivalent:

(1) $R$ is an $N R$ ring;

(2) $U_{n}(R)$ is an $N R$ ring;

(3) $L_{n}(R)$ is an $N R$ ring.

Proof. $(1) \Rightarrow(2)$ : Suppose that $R$ is $N R$. By observing that

$$
N\left(U_{n}(R)\right)=\left(\begin{array}{cccc}
N(R) & R & \cdots & R \\
0 & N(R) & \cdots & R \\
\vdots & \vdots & \ddots & \vdots \\
0 & 0 & \cdots & N(R)
\end{array}\right)
$$

the $\operatorname{ring} U_{n}(R)$ is also $N R$.

$(2) \Rightarrow(1)$ : Suppose that $U_{n}(R)$ is an $N R$ ring for any $n \geq 2$. Since $R$ is isomorphic to $\left\{a I_{n} \in U_{n}(R) \mid a \in R\right\}, R$ is $N R$ by Lemma 1.3(1) when $U_{n}(R)$ is $N R$, where $I_{n}$ is the $n$ by $n$ identity matrix.

The proofs of $(1) \Rightarrow(3)$ and $(3) \Rightarrow(1)$ are similar to those of $(1) \Rightarrow(2)$ and $(2) \Rightarrow(1)$.

However $\operatorname{Mat}_{n}(A)(n \geq 2)$ is not $N R$ by Lemma 1.3(2) for any ring $A$.

Proposition 2.2. Let $R, S$ be rings and ${ }_{R} M_{S}$ an $(R, S)$-bimodule. Let $E=$ $\left(\begin{array}{cc}R & M \\ 0 & S\end{array}\right)$. Then $E$ is $N R$ if and only if $R$ and $S$ are both $N R$.

Proof. By observing $N(E)=\left(\begin{array}{cc}N(R) & M \\ 0 & N(S)\end{array}\right)$, a similar proof to Theorem 2.1 yields the proposition.

From now on, $\mathbb{Z}$ denotes the ring of integers.

Proposition 2.3. (1) The class of $N R$ rings is closed under direct limits.

(2) $A$ direct sum of $N R$ rings $R_{i}(i \in I)$ is $N R$ if and only if $R_{i}$ is $N R$ for all $i \in I$.

(3) Let $S$ be an NR ring (possibly without identity) and attach an identity to $S$, obtaining $R=S \oplus \mathbb{Z}$. Then $R$ is $N R$. 
Proof. (1) Let $D=\left\{R_{i}, \alpha_{i j}\right\}$ be a direct system of $N R \operatorname{rings} R_{i}$ for $i \in I$ and ring homomorphisms $\alpha_{i j}: R_{i} \rightarrow R_{j}$ for each $i \leq j$ satisfying $\alpha_{i j}(1)=1$, where $I$ is a directed partially ordered set. Set $R=\underline{\lim } R_{i}$ the direct limit of $D$ with $\iota_{i}: R_{i} \rightarrow R$ and $\iota_{j} \alpha_{i j}=\iota_{i}$. Let $a, b \in \vec{R}$. Then $a=\iota_{i}\left(a_{i}\right)$, $b=\iota_{j}\left(b_{j}\right)$ for some $i, j \in I$ and there is $k \in I$ such that $i \leq k, j \leq k$. Define $a+b=\iota_{k}\left(\alpha_{i k}\left(a_{i}\right)+\alpha_{j k}\left(b_{j}\right)\right)$ and $a b=\iota_{k}\left(\alpha_{i k}\left(a_{i}\right) \alpha_{j k}\left(b_{j}\right)\right)$, where $\alpha_{i k}\left(a_{i}\right)$ and $\alpha_{j k}\left(b_{j}\right)$ are in $R_{k}$. Then $R$ forms a ring with $0=\iota_{i}(0)$ and $1=\iota_{i}(1)$.

Next let $a, b \in N(R)$. There is $k \in I$ such that $a, b \in N\left(R_{k}\right)$ via $\iota_{i}$ 's and $\alpha_{i j}$ 's. Since $R_{k}$ is $N R, a-b \in N\left(R_{k}\right)$ and $a b \in N\left(R_{k}\right)$, concluding $R$ being $N R$.

(2) Let $R=\sum_{i \in I} R_{i}$ and $a, b \in N(R)$ with $a=\left(a_{i}\right)$ and $b=\left(b_{i}\right)$. Here we can assume $a, b$ are both nonzero. Put $J=\left\{j \in I \quad a_{j} \neq 0\right.$ or $\left.b_{j} \neq 0\right\}$. Then clearly $J$ is finite. If all $R_{i}$ 's are $N R$, then $a_{j}-b_{j}, a_{j} b_{j} \in N\left(R_{j}\right)$ for all $j \in J$. Thus $a-b, a b \in N(R)$ since $J$ is finite, obtaining that $R$ is $N R$.

Conversely let $R$ be $N R$ and assume on the contrary that $R_{i_{0}}$ is not $N R$ for some $i_{0} \in I$. Then for some $x, y \in N\left(R_{i_{0}}\right), x-y \notin N\left(R_{i_{0}}\right)$ or $x y \notin N\left(R_{i_{0}}\right)$. Taking $a=\left(a_{i}\right), b=\left(b_{i}\right) \in R$ such that $x=a_{i_{0}}, y=b_{i_{0}}$ and $a_{k}=0, b_{k}=0$ for all $k \in I \backslash\left\{i_{o}\right\}$. Then since $a, b \in N(R)$ and $R$ is $N R, a-b \in N(R)$ and $a b \in N(R)$ and so $a_{i_{0}}-b_{i_{0}} \in N\left(R_{i_{0}}\right)$ and $a_{i_{0}} b_{i_{0}} \in N\left(R_{i_{0}}\right)$, a contradiction.

(3) Since $S$ is a subring of $R$ and $N(S) \oplus 0=N(R), R$ is $N R$.

Letting $I=\left\{M \in U_{n}(R) \mid\right.$ the diagonal entries of $M$ are all zero $\}$ in Theorem 2.1, $I$ is a nil ideal of $U_{n}(R)$ such that $U_{n}(R) / I$ is isomorphic to the direct sum of $n$-copies of $R$. So if $R$ is $N R$, then $U_{n}(R) / I$ in $N R$ by Proposition 2.3(2). Thus Lemma 1.3(5) implies that $U_{n}(R)$ is $N R$.

By Proposition 2.3(2), one may suspect that the direct product of $N R$ rings is $N R$. However the direct product of $N R$ rings need not be $N R$ as follows.

Example 2.4. The construction and computation are according to Huh et al. [19, Examples 1.6], [13, Example 2.5], and Marks [21, Remark, p. 508]. Let $K$ be a field and define $D_{n}=K\left\{x_{n}\right\}$, a free algebra generated by $x_{n}$, with a relation $x_{n}^{n+2}=0$ for each nonnegative integer $n$. Then clearly $D_{n} \cong$ $K[x] /\left(x^{n+2}\right)$, where $\left(x^{n+2}\right)$ is the ideal of $K[x]$ generated by $x^{n+2}$. Next let $R_{n}=\left(\begin{array}{cc}D_{n} & x_{n} D_{n} \\ x_{n} D_{n} & D_{n}\end{array}\right)$ be a subring of the 2 by 2 matrix ring over $D_{n}$. Then every $R_{n}$ is 2-primal (hence $N R$ ) by the computation in [19, Example 1.6]. Set $R=\prod_{n=0}^{\infty} R_{n}$. However the sum of two nilpotent elements $\left(\begin{array}{cc}0 & x_{n} \\ 0 & 0\end{array}\right)$ and $\left(\begin{array}{cc}0 & 0 \\ x_{n} & 0\end{array}\right)$ is not nilpotent, entailing that $R$ is not $N R$.

Proposition 2.5. Let e be a central idempotent of a ring $R$. Then the following statements are equivalent:

(1) $R$ is NR;

(2) $e R$ and $(1-e) R$ are both $N R$.

Proof. $(1) \Rightarrow(2)$ comes from Lemma 1.3(1) since $e R$ and $(1-e) R$ are subrings of $R$. 
$(2) \Rightarrow(1)$ : Suppose that $e R$ and $(1-e) R$ are both $N R$. Then by observing $N(R)=N(e R) \oplus N((1-e) R)$, Proposition 2.3(2) implies that $R$ is $N R$.

The polynomial rings need not preserve the $N R$ condition as follows.

Example 2.6. The following construction is due to Smoktunowicz [25]. Let $K$ be a countable field and $\bar{A}$ the algebra of polynomials with zero constant terms in non-commuting indeterminates $x, y, z$ over $K$. Then $\bar{A}$ can be enumerated, say $\bar{A}=\left\{f_{1}, f_{2}, \ldots\right\}$. By the argument in the proof of $[25$, Theorem 12$]$, there are natural numbers $m_{1}, m_{2}, \ldots$ such that (i) $m_{1}>10^{8}, m_{i+1}>m_{i} 2^{i+101}$ for $i \geq 1$, (ii) each $m_{i}$ divides $m_{i+1}$ and (iii) $m_{i}>3^{2 \operatorname{deg}\left(f_{i}\right)}\left(\operatorname{deg}\left(f_{i}\right)\right)^{2} 40^{2}$ for $i \geq 1$. Let $I$ be the ideal of $\bar{A}$ generated by $\left\{f_{i}^{10 m_{i+1}} \mid i=1,2, \ldots\right\}$ and $S=\bar{A} / I$. Then $S$ is nil. Attach an identity to $S$, obtaining $R=S \oplus \mathbb{Z} . N(R)=S \oplus 0$ implies that $R$ is $N I$ (hence $N R$ ).

Next assume that the polynomial ring over an $N R$ ring is also $N R$. Then the polynomial ring $R[X, Y]$ in two commuting indeterminates $X, Y$ over $R$ is also $N R$. However $\bar{x}+\bar{y} X+\bar{z} Y$ is not nilpotent by the proof of $[25$, Theorem $12]$, in spite of $\bar{x}, \bar{y} X, \bar{z} Y \in N(R[X, Y])$. This is contrary to that $R[X, Y]$ is $N R$.

Suppose that an $N I \operatorname{ring} R$ is of bounded index of nilpotency. Then $R$ is 2-primal by [13, Proposition 1.4] and moreover $R[x]$ is 2-primal (hence $N R$ ) by [5, Proposition 2.6].

Next we consider the $N R$ condition of Ore extensions. For a ring $R$, a ring endomorphism $\sigma: R \rightarrow R$ and a $\sigma$-derivation $\delta: R \rightarrow R$, the Ore extension $R[x ; \sigma, \delta]$ of $R$ is the ring obtained by giving $R[x]$ the multiplication $x r=$ $\sigma(r) x+\delta(r)$ for all $r \in R$. If $\delta=0$, we write $R[x ; \sigma]$ for $R[x ; \sigma, 0]$ and is called an Ore extension of endomorphism type (also called a skew polynomial ring). While if $\sigma=1$, we write $R[x ; \delta]$ for $R[x ; 1, \delta]$ and is called an Ore extension of derivation type (also called a differential polynomial ring). Use $\mathbb{Z}_{n}$ to denote the ring of integers modulo $n$.

Example 2.7. (1) There exists an $N R$ ring over which the skew polynomial ring need not be $N R$. For a domain $D$ let $R=D \oplus D$, then $R$ is reduced (hence $N R$ ). Consider the automorphism $\sigma$ of $R$ defined by $\sigma(s, t)=(t, s)$. Let $R[x ; \sigma]$ be the skew polynomial ring over $R$ by $\sigma$. Consider $(1,0) x$ and $(0,1) x$. Then $((1,0) x(1,0) x)=0$ and $((0,1) x(0,1) x)=0$, whence $(1,0) x,(1,0) x \in$ $N(R[x ; \sigma])$. But $(1,0) x+(0,1) x=(1,1) x$ is not nilpotent, entailing that $R[x ; \sigma]$ is not $N R$.

(2) There exists an $N R$ ring over which the differential polynomial ring need not be $N R$. We use the ring and argument in [4, Example 11]. Let $\mathbb{Z}_{2}[t]$ be the polynomial ring with an indeterminate $t$ over $\mathbb{Z}_{2}$. Then $R=\mathbb{Z}_{2}[t] /\left(t^{2}\right)$ is commutative (hence $N R$ ), where $\left(t^{2}\right)$ is the ideal of $\mathbb{Z}_{2}[t]$ generated by $t^{2}$. Define a derivation $\delta$ on $R$ by $\delta\left(t+\left(t^{2}\right)\right)=1+\left(t^{2}\right)$. Then $R[x ; \delta] \cong \operatorname{Mat}_{2}\left(\mathbb{Z}_{2}\left[y^{2}\right]\right)$ which is not $N R$ by Lemma $1.3(2)$. 
In the following we see two cases in which $R[x ; \sigma, \delta]$ can be $N R$.

Let $\sigma$ be an endomorphism of a ring $R$. Due to Krempa [16], $\sigma$ is called rigid if $r \sigma(r)=0$ implies $r=0$ for $r \in R$. It is easily checked that a rigid endomorphism is injective. A ring $R$ is called $\sigma$-rigid if there exists a rigid endomorphism $\sigma$ of R. Hong et al. [11, Proposition 5] proved that a ring R is $\sigma$-rigid if and only if $R[x ; \sigma, \delta]$ is a reduced ring and $\sigma$ is a monomorphism of $R$. In Example 2.7(1), $\sigma$ is not rigid since $(1,0) \sigma(1,0)=0$.

Let $R$ be a local ring with an endomorphism $\sigma$ and a $\sigma$-derivation $\delta$, and suppose that the maximal ideal $M$ of $R$ is nilpotent. In this situation, Mark [20, Theorem 3.4] proved that $R[x ; \sigma, \delta]$ is 2-primal if $\delta(M) \subseteq M$. In Example $2.7(2), R$ is a local ring with the maximal ideal $M=\mathbb{Z}_{2} t+\left(t^{2}\right)$, but $\delta(M) \nsubseteq M$ since $\delta(M)$ contains $1+\left(t^{2}\right)$.

Next we study the $N R$ condition of classical quotient rings over $N R$ rings. A ring $R$ is called right Ore if given $a, b \in R$ with $b \in C(0)$ there exist $a_{1}, b_{1} \in R$ with $b_{1} \in C(0)$ such that $a b_{1}=b a_{1}$. Left Ore rings can be defined by symmetry. It is well-known that $R$ is a right Ore ring if and only if the classical right quotient ring of $R$ exists. If both right and left quotient rings exist, then they are equal. Let $F$ be a field and $R$ the free algebra in two indeterminates over $F$. Then $R$ is a domain but cannot be right (left) Ore. It is also well-known that $R$ is a right Ore domain if and only if the classical right quotient ring of $R$ is a division ring. A subset $I$ of a ring $R$ is called right (resp. left) T-nilpotent provided that for every sequence $a_{1}, a_{2}, \ldots$ in $I$ there is a positive integer $n$ such that $a_{n} \cdots a_{2} a_{1}=0$ (resp. $a_{1} a_{2} \cdots a_{n}=0$ ). Nilpotent subsets of a ring are obviously both right and left T-nilpotent but the converse does not hold in general, and that right or left T-nilpotent subsets are clearly nil but nil ideals need not be right (or left) T-nilpotent. The T-nilpotence is not left-right symmetric by [24, Example 2.7.38].

Theorem 2.8. Let $R$ be a right Ore ring and $Q$ the classical right quotient ring of $R$. Suppose that $N(R)$ is left T-nilpotent. Then the following conditions are equivalent:

(1) $Q$ is an $N I$ local ring with $N(Q)=J(Q)=\left\{a b^{-1} \in Q \mid a \in N(R)\right.$ and $b \in$ $C(0)\}$

(2) $R$ is $N R$ and $C(0)=C\left(N^{*}(R)\right)=R \backslash N(R)$.

Proof. $(1) \Rightarrow(2)$ : We will apply the proof of [8, Proposition 2.1(1)]. Suppose that the condition (1) holds. Then $R$ is $N I$ (hence $N R$ ) by Lemma 1.3(4) and $Q / N(Q)$ is a division ring. So if $a, b \in R \backslash N(R)$, then they are invertible in $Q / N(Q)$ and so $a b \notin N(Q)$. Thus $a b \notin N(R)$ since $N(R) \subseteq N(Q)$. This yields that $R / N(R)$ is a domain, and hence $C(N(R))=R \backslash N(R)$ and $C(0) \subseteq$ $C(N(R))$. Conversely let $u \in C(N(R))$. Then $u$ is invertible in $Q$ since $Q / N(Q)$ is a division ring with $N(Q)=J(Q)=\left\{a b^{-1} \in Q \mid a \in N(R)\right.$ and $\left.b \in C(0)\right\}$, entailing $u \in C(0)$. This yields $C(N(R)) \subseteq C(0)$.

$(2) \Rightarrow(1)$ : Assume that $R$ is $N R$ and $C(0)=R \backslash N(R)$. Then $R$ is $N I$ by Lemma 1.3(3) and moreover $R / N^{*}(R)$ is a domain. Next we will apply the 
proof of [8, Proposition 2.1(1)]. Let $a b^{-1} \in N(Q)$. Then $a$ is not regular, forcing $a \in N(R)$ by the assumption. Conversely let $a b^{-1} \in Q$ with $a \in N(R)$. Then since $C(0)=R \backslash N(R)$ we have

$$
\left(a b^{-1}\right)^{2}=a c_{1} d_{1}^{-1},\left(a b^{-1}\right)^{3}=a c_{1} c_{2} d_{2}^{-1}, \ldots,\left(a b^{-1}\right)^{n+1}=a c_{1} \cdots c_{n} d_{n}^{-1}
$$

with $c_{i}$ 's in $N(R)$ and $d_{i}$ 's in $C(0)$ for $i=1, \ldots, n$. But $N(R)$ is left Tnilpotent by hypothesis and so $\left(a b^{-1}\right)^{k}=a c_{1} \cdots c_{k-1} d_{k-1}^{-1}=0$ for some $k$. Whence $a b^{-1} \in N(Q)$ when $a \in N(R)$, entailing

$$
N(Q)=\left\{a b^{-1} \in Q \mid a \in N(R) \text { and } b \in C(0)\right\} .
$$

From $C(0)=R \backslash N(R), N(Q)$ is an ideal of $Q$ and so $Q$ is $N I$. Consequently $Q / N(Q)$ is a division ring since every element in $Q \backslash N(Q)$ is invertible. So $Q$ is a local ring such that $N(Q)=J(Q)=\left\{a b^{-1} \in Q \mid a \in N(R)\right.$ and $\left.b \in C(0)\right\}$.

Considering Theorem 2.8 and [8, Proposition 2.1], one may conjecture $N(R)$ $=N_{*}(R)$ under the equivalences of Theorem 2.8. However the following provides a counterexample.

Example 2.9. Let $S=\mathbb{Z}_{2}[x]$ and consider

$$
D_{n}(S)=\left\{M \in U_{n}(S) \mid \text { the diagonal entries of } M \text { are equal }\right\},
$$

a subring of $U_{n}(S)$ and define a map $\sigma: D_{n}(S) \rightarrow D_{n+1}(S)$ by $A \mapsto\left(\begin{array}{cc}A & 0 \\ 0 & A\end{array}\right)$. Then $D_{n}(S)$ can be considered as a subring of $D_{n+1}(S)$ via $\sigma$ (i.e., $A=\sigma(A)$ for $\left.A \in D_{n}(S)\right)$. Set $R$ be the direct limit of the direct system $\left(D_{n}(S), \sigma_{i j}\right)$ with $\sigma_{i j}=\sigma^{j-i}$. Then $R$ is semiprime (i.e., $N_{*}(R)=0$ ) by [14, Theorem 2.2(2)] but $N^{*}(R) \neq 0$ (hence not 2-primal). Moreover $R$ is $N I$ by [13, Example 1.2] and Lemma 1.3(4). Note

$$
N(R)=\{M \in R \mid \text { the diagonal entries of } M \text { are zero }\} \neq 0
$$

and so

$$
\begin{aligned}
C(0)=C(N(R)) & =R \backslash N(R) \\
& =\{M \in R \mid \text { the diagonal entries of } M \text { are nonzero }\} .
\end{aligned}
$$

By [17, Theorem 1.3 and Proposition 1.9], every $D_{n}(S)$ is both left and right Ore; hence $R$ is also left and right Ore by the construction. It is easily checked that the classical quotient $\operatorname{ring} Q$ of $R$ is the direct limit of the direct system $\left(D_{n}\left(\mathbb{Z}_{2}(x)\right), \sigma_{i j}\right)$ with $\sigma_{i j}=\sigma^{j-i}$, where $\mathbb{Z}_{2}(x)$ is the quotient field of $\mathbb{Z}_{2}[x]$. Note that $Q$ is a $\pi$-regular ring with

$$
N(Q)=J(Q)=\{N \in Q \mid \text { the diagonal entries of } N \text { are zero }\} \neq 0 .
$$

This result yields that $Q$ is $N I$ and local with

$$
N(Q)=J(Q)=\left\{a b^{-1} \in Q \mid a \in N(R) \text { and } b \in C(0)\right\} .
$$

Notice that $N(R)$ is left T-nilpotent. Indeed, consider a sequence $a_{1}, a_{2}, \ldots$ in $N(R)$. We can assume that every $a_{i}=\left(a_{s t}\right)_{i}$ is nonzero. Let $j$ be largest such 
that the $j$-th row of $a_{1}$ contains a nonzero entry. Then since $s<t$ in each $a_{i}$, we get $a_{j+1} a_{j} \cdots a_{2} a_{1}=0$.

If given a ring $R$ is $\pi$-regular, then every regular element is invertible and so $R$ is itself its right (left) quotient ring. Thus letting $S$ be any division ring in Example 2.9, the direct limit $R$ and its classical right (left) quotient ring coincide since $R$ is $\pi$-regular. This provides another counterexample since $N(R) \neq 0$ and $N_{*}(R)=0$ by a similar computation.

In Theorem 2.8, each equality in $C(0)=C\left(N^{*}(R)\right)=R \backslash N(R)$ is necessary as the following examples show.

Example 2.10. The argument is almost all due to [8, Example 2.2]. (1) (The case of $\left.C(0)=C\left(N^{*}(R)\right) \varsubsetneqq R \backslash N(R)\right)$ We refer the argument in [8, Example $2.2(1)]$. Let $R=U_{2}(\mathbb{Z})$. Then clearly $N(R)=N^{*}(R)=\left(\begin{array}{cc}0 & \mathbb{Z} \\ 0 & 0\end{array}\right)$; and the classical right quotient ring $Q$ of $R$ is $U_{2}(\mathbb{Q})$, where $\mathbb{Q}$ is the field of rational numbers. $Q$ is $N I$ with $N(Q)=J(Q)=\left\{a b^{-1} \in Q \mid a \in N(R)\right.$ and $\left.b \in C(0)\right\}=\left(\begin{array}{ll}0 & \mathbb{Q} \\ 0 & 0\end{array}\right)$. However $Q$ is not local since $Q / J(Q) \cong \mathbb{Q} \oplus \mathbb{Q}$, and $C(0)=C(N(R))=$ $\left\{\left(\begin{array}{ll}a & b \\ 0 & c\end{array}\right) \in R \mid a \neq 0, c \neq 0\right\} \varsubsetneqq R \backslash N(R)=\left\{\left(\begin{array}{ll}a & b \\ 0 & c\end{array}\right) \in R \mid a \neq 0\right.$ or $\left.c \neq 0\right\}$.

(2) (The case of $C(0) \varsubsetneqq C\left(N^{*}(R)\right)=R \backslash N(R)$ ) We refer the argument in $\left[8\right.$, Example 2.2(2)]. Let $R=\left\{\left(\begin{array}{cc}n & a \\ 0 & n\end{array}\right) \mid n \in \mathbb{Z}\right.$ and $\left.a \in \mathbb{Z} / 2 \mathbb{Z}\right\}$. Then $R$ is commutative, $C(0)=\left\{\left(\begin{array}{cc}m & a \\ 0 & m\end{array}\right) \in R \mid m\right.$ is odd $\}$, and $C(N(R))=R \backslash N(R)=$ $\left\{\left(\begin{array}{ll}s & a \\ 0 & s\end{array}\right) \in R \mid s \neq 0\right\}$ because $N(R)=\left(\begin{array}{cc}0 & \mathbb{Z} / 2 \mathbb{Z} \\ 0 & 0\end{array}\right)$ and $R / N(R) \cong \mathbb{Z}$. The classical right quotient ring $Q$ of $R$ is

$$
\left\{\left(\begin{array}{ll}
q & a \\
0 & q
\end{array}\right) \mid q \in \mathbb{Z}_{(2)}, a \in \mathbb{Z}_{2}\right\},
$$

where $\mathbb{Z}_{(2)}$ denotes the localization of $\mathbb{Z}$ at the prime ideal $2 \mathbb{Z}$. Then $Q$ is a commutative local ring with $N(Q)=\left\{a b^{-1} \in Q \mid a \in N(R)\right.$ and $\left.b \in C(0)\right\}=$ $\left(\begin{array}{cc}0 & \mathbb{Z} / 2 \mathbb{Z} \\ 0 & 0\end{array}\right) \varsubsetneqq J(Q)=\left\{\left(\begin{array}{cc}q & a \\ 0 & q\end{array}\right) \in Q \mid q \in J\left(\mathbb{Z}_{(2)}\right)\right\}$. Note that $Q / N(Q) \cong \mathbb{Z}_{(2)}$ and $Q / J(Q) \cong \mathbb{Z} / 2 \mathbb{Z}$

(3) (The case of $\left.C(0) \varsubsetneqq C\left(N^{*}(R)\right) \varsubsetneqq R \backslash N(R)\right)$. We refer the argument in [8, Example 2.2(3)]. We use the ring and the argument in [5, Example 5.10]. Let

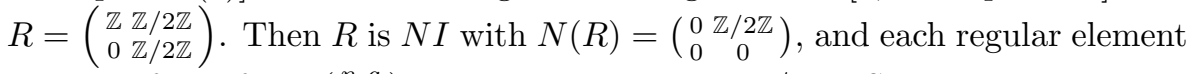
in $R$ is of the form $\left(\begin{array}{ll}n & a \\ 0 & \frac{1}{1}\end{array}\right)$ with $n$ odd and $a \in \mathbb{Z} / 2 \mathbb{Z}$. So the classical right quotient ring $Q$ of $R$ is $\left(\begin{array}{cc}\mathbb{Z}_{(2)} & \mathbb{Z} / 2 \mathbb{Z} \\ 0 & \mathbb{Z} / 2 \mathbb{Z}\end{array}\right) . Q$ is clearly $N I$. However $Q$ is not local since $Q / J(Q) \cong \mathbb{Z} / 2 \mathbb{Z} \oplus \mathbb{Z} / 2 \mathbb{Z}$, and $N(Q)=\left\{a b^{-1} \in Q \mid a \in N(R)\right.$ and $b \in$ $C(0)\}=\left(\begin{array}{ll}0 & F \\ 0 & 0\end{array}\right) \varsubsetneqq J(Q)=\left(\begin{array}{cc}J\left(\mathbb{Z}_{(2)}\right) & \mathbb{Z} / 2 \mathbb{Z} \\ 0 & 0\end{array}\right)$. Note that every element of $R$ with $(1,1)$-entry even is not regular, and this yields

$$
\begin{aligned}
C(0) & =\left\{\left(\begin{array}{cc}
m & a \\
0 & b
\end{array}\right) \in R \mid m \text { is odd and } b=\overline{1}\right\} \\
& \varsubsetneqq C(N(R))=\left\{\left(\begin{array}{cc}
k & a \\
0 & b
\end{array}\right) \in R \mid k \neq 0 \text { and } b \neq 0\right\} \\
& \varsubsetneqq R \backslash N(R)=\left\{\left(\begin{array}{cc}
h & a \\
0 & b
\end{array}\right) \in R \mid h \neq 0 \text { or } b \neq 0\right\} .
\end{aligned}
$$




\section{References}

[1] D. D. Anderson and V. Camillo, Armendariz rings and Gaussian rings, Comm. Algebra 26 (1998), no. 7, 2265-2272.

[2] R. Antoine, Nilpotent elements and Armendariz rings, J. Algebra 319 (2008), no. 8, 3128-3140.

[3] E. P. Armendariz, A note on extensions of Baer and P.P.-rings, J. Austral. Math. Soc. 18 (1974), 470-473.

[4] E. P. Armendariz, H. K. Koo, and J. K. Park, Isomorphic Ore extensions, Comm. Algebra 15 (1987), no. 12, 2633-2652.

[5] G. F. Birkenmeier, H. E. Heatherly, and E. K. Lee, Completely prime ideals and associated radicals, (English summary) Ring theory (Granville, OH, 1992), 102-129, World Sci. Publ., River Edge, NJ, 1993.

[6] G. F. Birkenmeier, J. Y. Kim, and J. K. Park, Regularity conditions and the simplicity of prime factor rings, J. Pure Appl. Algebra 115 (1997), no. 3, 213-230.

[7] V. Camillo and P. P. Nielsen, McCoy rings and zero-divisors, J. Pure Appl. Algebra 212 (2008), no. 3, 599-615.

[8] Y. U. Cho, N. K. Kim, M. H. Kwon, and Y. Lee, Classical quotient rings and ordinary extensions of 2-primal rings, Algebra Colloq. 13 (2006), no. 3, 513-523.

[9] K. R. Goodearl, Von Neumann Regular Rings, Monographs and Studies in Mathematics, 4. Pitman (Advanced Publishing Program), Boston, Mass.-London, 1979.

[10] K. R. Goodearl and R. B. Warfield, Jr., An Introduction to Noncommutative Noetherian Rings, London Mathematical Society Student Texts, 16. Cambridge University Press, Cambridge, 1989.

[11] C. Y. Hong, N. K. Kim, and T. K. Kwak, Ore extensions of Baer and p.p.-rings, J. Pure Appl. Algebra 151 (2000), no. 3, 215-226.

[12] C. Huh, Y. Lee, and A. Smoktunowicz, Armendariz rings and semicommutative rings, Comm. Algebra 30 (2002), no. 2, 751-761.

[13] S. U. Hwang, Y. C, Jeon, and Y. Lee, Structure and topological conditions of NI rings, J. Algebra 302 (2006), no. 1, 186-199.

[14] Y. C. Jeon, H. K. Kim, Y. Lee, and J. S. Yoon, On weak Armendariz rings, Bull. Korean Math. Soc. 46 (2009), no. 1, 135-146.

[15] N. K. Kim and Y. Lee, Nilideals and nil-Armendariz rings, preprint.

[16] J. Krempa, Some examples of reduced rings, Algebra Colloq. 3 (1996), no. 4, 289-300

[17] C. I. Lee and Y. Lee, Properties and related conditions of K-rings, preprint.

[18] T.-K. Lee and T.-L. Wong, On Armendariz rings, Houston J. Math. 29 (2003), no. 3, 583-593.

[19] Y. Lee, Y. Lee, and H. K. Kim, Questions on 2-primal rings, Comm. Algebra 26 (1998), no. 2, 595-600.

[20] G. Marks, On 2-primal Ore extensions, Comm. Algebra 29 (2001), no. 5, 2113-2123.

[21] _ A taxonomy of 2-primal rings, J. Algebra 266 (2003), no. 2, 494-520.

[22] M. Morse and G. A. Hedlund, Symbolic dynamics, Amer. J. Math. 60 (1936), 815-866.

[23] M. B. Rege and S. Chhawchharia, Armendariz rings, Proc. Japan Acad. Ser. A Math. Sci. 73 (1997), no. 1, 14-17.

[24] L. H. Rowen, Ring Theory, Academic Press, Inc., San Diego, 1991.

[25] A. Smoktunowicz, Polynomial rings over nil rings need not be nil, J. Algebra 233 (2000), no. $2,427-436$.

YOUNG CHUN

Department of Mathematics

Korea SCIENCE ACADEMY

Busan 614-103, Korea

E-mail address: ch3746@nate.com 
Young Cheol Jeon

Department of Mathematics

Korea Science Academy

BUSAN 614-103, KOREA

E-mail address: jachun@chol.com

SUNGKYUNG KANG

Department of Mathematics

Korea Science Academy

Busan 614-103, Korea

E-mail address: sungkyung38@naver.com

Key Nyoung Lee

Department of Mathematics

Korea Science Academy

Busan 614-103, Korea

E-mail address: key2364@hanmail.net

YANG LEE

Department of Mathematics Education

Pusan National University

BUSAN 609-735, Korea

E-mail address: ylee@pusan.ac.kr 